\title{
Low-Cost, High-Performance Fiber Optic Fabry-Perot Sensor for Ultrasonic Wave Detection
}

\author{
Haoyong $\mathrm{Li}^{1,2}$, Delin $\mathrm{Li}^{1,2}$, Chaoyu Xiong ${ }^{1,2}$, Wenrong $\mathrm{Si}^{3}{ }^{\circledR}$, Chenzhao $\mathrm{Fu}^{3}$, Peng Yuan ${ }^{4}$ and \\ Yiting $\mathrm{Yu}^{1,2, *}$ \\ 1 Key Laboratory of Micro/Nano Systems for Aerospace (Ministry of Education), Northwestern Polytechnical \\ University, Xi'an 710072, China; lyhaoyong@mail.nwpu.edu.cn (H.L.); delinli@mail.nwpu.edu.cn (D.L.); \\ bearluckyyeah@163.com (C.X.) \\ 2 Key Laboratory of Micro- and Nano-Electro-Mechanical Systems of Shaanxi Province, \\ Northwestern Polytechnical University, Xi'an 710072, China \\ 3 State Grid Shanghai Electric Power Research Institute, Shanghai 200437, China; siwenrong@126.com (W.S.); \\ 13512111246@139.com (C.F.) \\ 4 Xi'an Maorong Power Equipment Co., Ltd., Xi'an 710048, China; y5anpeng@126.com \\ * Correspondence: yyt@nwpu.edu.cn; Tel.: +86-29-88460353
}

Received: 17 December 2018; Accepted: 16 January 2019; Published: 19 January 2019

check for updates

\begin{abstract}
This study describes a novel fiber optic extrinsic Fabry-Perot interferometric (EFPI) ultrasonic sensor comprising a low-cost and high-performance silicon diaphragm. A vibrating diaphragm, $5 \mu \mathrm{m}$ thick, was fabricated by using the Microelectromechanical Systems (MEMS) processing technology on a silicon-on-insulator (SOI) wafer. The Fabry-Perot (FP) cavity length was solely determined during the manufacturing process of the diaphragm by defining a specific stepped hole on the handling layer of the SOI wafer, which made the assembly of the sensor easier. In addition, the use of cheap and commercially available components and MEMS processing technology in the development of the sensing system, limited the cost of the sensor. The experimental tests showed that the minimum detectable ultrasonic pressure was $1.5 \mathrm{mPa} / \mathrm{sqrt}(\mathrm{Hz})-0.625 \mathrm{mPa} / \mathrm{sqrt}(\mathrm{Hz})$ between $20 \mathrm{kHz}$ and $40 \mathrm{kHz}$. As a result, this sensor has the potential to successfully detect weak ultrasonic signals.
\end{abstract}

Keywords: ultrasonic pressure detection; Fabry-Perot; fiber optic sensors

\section{Introduction}

Ultrasonic waves (frequency $>20 \mathrm{kHz}$ ) have extensive applications in diverse areas, such as detection of defects in various materials and structures, sonar detection, medical diagnostics, etc. In these applications, ultrasonic sensors always play a vital role in acquiring ultrasonic wave information. The fiber optic extrinsic Fabry-Perot interferometric (EFPI) sensors have a number of inherent advantages such as; high-frequency response, immunity to electromagnetic interference (EMI), remote sensing capability, small size, light weight and easy construction; therefore, they have become an ideal candidate for ultrasonic detection [1-5].

The diaphragm is one of the core components of optical fiber EFPI sensors. To ensure that it has an efficient frequency response to ultrasonic waves, the diaphragm is generally designed to have a high natural frequency, which results in a low sensitivity. For this reason, the trade-off between the natural frequency and the sensitivity must be considered, and the material and dimension of a diaphragm need to be optimized in order to obtain a desired sensitivity as well as the required natural frequency [6]. Wonuk Jo et al. fabricated an EFPI acoustic sensor using a photonic-crystal silicon diaphragm with a thickness of $450 \mathrm{~nm}$. The sensor showed very high sensitivity and excellent 
minimal detectable pressure, but its maximal detectable acoustic frequency was relatively low [2]. Lijun Song et al. proposed an optical fiber EFPI ultrasonic sensor whose $150 \mu \mathrm{m}$-thick fused silicon diaphragm can detect over $100 \mathrm{kHz}$ ultrasonic waves, but its sensitivity is greatly reduced [7].

At present, there are numerous methods to construct a Fabry-Perot (FP) cavity on the end surface of an optical fiber [6-10]. The traditional method consists in adjusting the distance between the fiber and the diaphragm by using a precise positioning platform under the continuous monitoring of a spectrometer and in performing bonding packaging when the cavity length is at the working point [8]. This method can assemble the FP cavity precisely but it is inefficient. Another common method involves adjusting the central wavelength of the light source by an optical filter to ensure the desired FP cavity length [9]. Currently, researchers tend to determine the FP cavity length during diaphragm fabrication in order to improve the efficiency of assembly [10-14]. E. Cibula et al. proposed a new all-glass fiber EFPI pressure sensor. An air cavity on the fiber was formed firstly by an etching process, and then the air cavity was fused with a lead-in fiber to form the FP cavity [10]. The Stanford group described an EFPI acoustic sensor, where the length between the optical fiber and the diaphragm was controlled by attaching two thin spacers to the fixed fiber capillary [11]. These methods can realize the precise assembly of the FP cavity on optical fiber. However, it is urgent to further facilitate sensor assembly and simplify the process. In addition, in most of the reported work, the optical fiber must be preprocessed and fixed by capillaries before sensor assembly, which remains a barrier for the mass production of the optical fiber EFPI sensors [8-12].

In this research, the vibrating diaphragm of a sensor with a thickness of $5 \mu \mathrm{m}$ was fabricated by employing the Microelectromechanical Systems (MEMS) processing technology on a silicon-on-insulator (SOI) wafer. A stepped hole was designed on the handling layer of the SOI wafer to define the FP cavity when manufacturing the diaphragm, which made the sensor assembly easier. In addition, a commercially available optical fiber patch cord (PC) was directly employed to assemble the FP cavity without any follow-up processing. The experimental test showed that the minimum detectable ultrasonic pressure of the sensor was from $1.5 \mathrm{mPa} / \operatorname{sqrt}(\mathrm{Hz})$ to $0.625 \mathrm{mPa} / \operatorname{sqrt}(\mathrm{Hz})$ between $20 \mathrm{kHz}$ and $40 \mathrm{kHz}$.

\section{Design and Fabrication}

\subsection{Structural Design}

The EFPI sensor was interrogated by using the quadrature detection technique [15], which uses a laser source at a fixed wavelength and a photodetector, as shown in Figure 1.

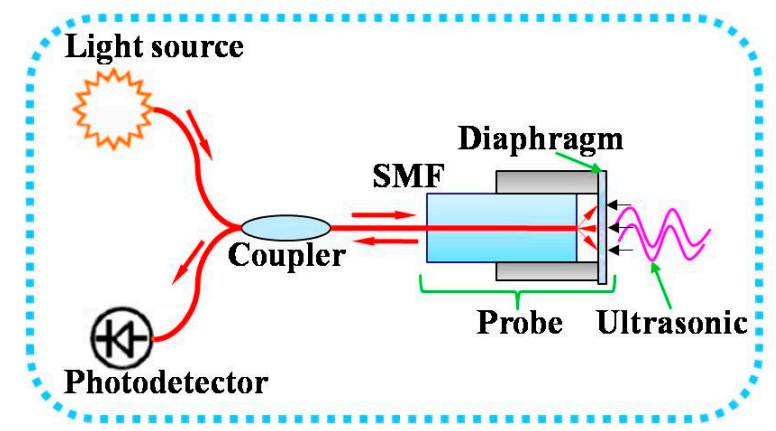

Figure 1. Schematic illustration of the fiber optic extrinsic Fabry-Perot interferometric (EFPI) ultrasonic sensor.

The ultrasonic wave is a dynamic excitation source. To ensure the response of the EFPI sensor to the ultrasonic wave, the natural frequency $f$ of the vibrating diaphragm must be determined first 
during the design, and that of the circle diaphragm can be expressed by Equation (1), according to reference [16]:

$$
f=\frac{a h}{4 \pi R^{2}} \sqrt{\frac{E}{3 \rho\left(1-\mu^{2}\right)}}
$$

where $a$ is the coefficient of vibration mode, $\rho, E, \mu, h$, and $R$ are density, elastic modulus, Poisson's ratio, thickness, and radius of the diaphragm, respectively.

After the natural frequency was determined, the larger central deformation $y(p)$ of the diaphragm corresponded to the higher sensitivity under the same acoustic pressure $p$. Since the ultrasonic wave is a dynamic signal, the diaphragm deformation depends on the damping ratio and its stiffness. In practice, static pressure is usually applied to replace the dynamic pressure during diaphragm design, since the damping ratio and stiffness are difficult to determine. When the static pressure $p$ is applied on the circle diaphragm, its central deformation $y(p)$ can be obtained by Equation (2) [16]:

$$
y(p)=\frac{3\left(1-\mu^{2}\right) p}{16 E h^{3}} R^{4}
$$

As a result, after the natural frequency of the diaphragm that is necessary to achieve the maximal deformation has been determined, only the material and geometrical parameters $(R$ and $h)$ of the diaphragm need to be optimized.

If the material and the natural frequency of the diaphragm are determined, the ratio $h / R^{2}$ is a constant according to Equation (1), and thus $R$ and $h$ must increase or decrease at the same time to determine the maximum diaphragm deformation. However, referring to Equation (2), when $R$ and $h$ increase or decrease at the same time, the change of diaphragm deformation $y(p)$ is still uncertain. For the purpose of solving the above problem, we can multiply Equations (1) and (2), obtaining Equation (3):

$$
y(p)=\frac{1}{f} \cdot \frac{a h}{4 \pi R^{2}} \sqrt{\frac{E}{3 \rho\left(1-\mu^{2}\right)}} \cdot \frac{3\left(1-\mu^{2}\right) p}{16 E h^{3}} R^{4}=\frac{a p}{4 \pi f} \cdot \sqrt{\frac{E}{3 \rho\left(1-\mu^{2}\right)}} \cdot \frac{3\left(1-\mu^{2}\right)}{16 E} \cdot\left(\frac{R}{h}\right)^{2}
$$

According to Equation (3), the conclusion can be drawn that when the material and the natural frequency of the diaphragm are determined, the larger the ratio of $R / h$ is, the greater the diaphragm deformation will be under the same acoustic pressure $p$. In our previous research, we concluded that once the natural frequency and the material of the diaphragm are fixed, the smaller the thickness $h_{i}$, the larger the $R / h$ ratio [16]. In other words, if the natural frequency of the diaphragm has been determined, a thinner diaphragm will result in a larger deformation.

However, by calculating the natural frequency of the diaphragm versus the thickness $h$ and the radius $R$ according to Equation (1), as shown in Figure 2a, we found that when the natural frequency of the diaphragm is invariable, the radius $R$ decreases rapidly with the decreasing thickness $h$. When $R$ is too small, sensor assembly is very difficult. Moreover, for fiber optic EFPI sensors, the diaphragm size must be larger than that of the reflecting area for FP interference. This diameter can be calculated by the formula: $d_{\text {reflection }}=d_{\text {core }}+2 N_{A} L$ ( $d_{\text {reflection }}, L, d_{\text {core }}, N_{A}$ are the diameter of the reflecting area, the FP cavity length, the core's diameter, and the numerical aperture of the fiber optic, respectively), as denoted in Figure 2b. Therefore, although the deformation of the diaphragm will increase as the diaphragm thickness decreases, the diaphragm thickness cannot be reduced infinitely, considering the sensor assembly and the reflecting area. Currently, most of the reported silicon diaphragm are much larger than $20 \mu \mathrm{m}$, which is mainly attributed to the limitation of micromachining processes. Therefore, reducing the diaphragm thickness is still the primary way to improve the performance of fiber optic EFPI ultrasonic sensors. 


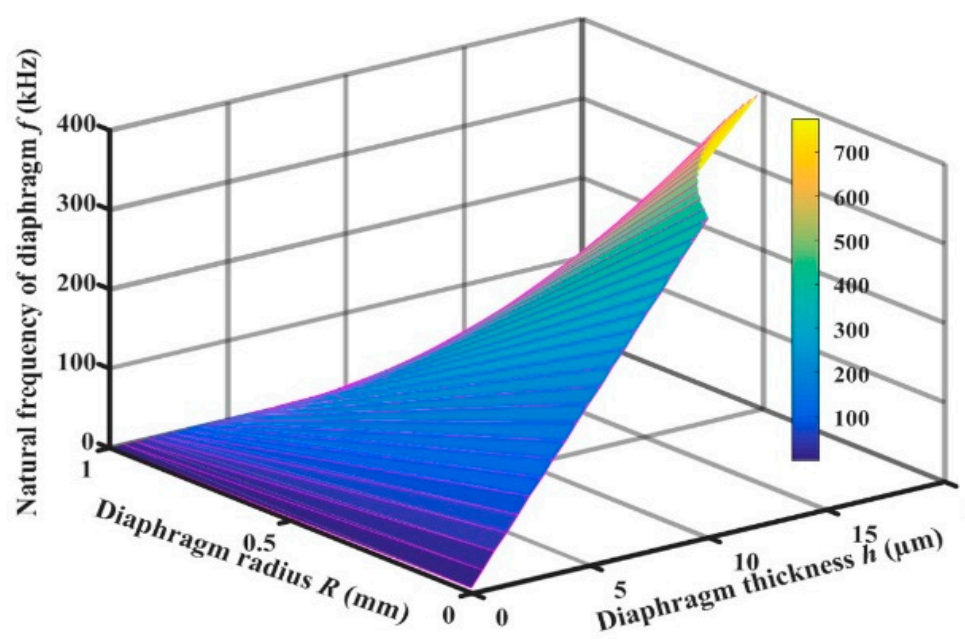

(a)

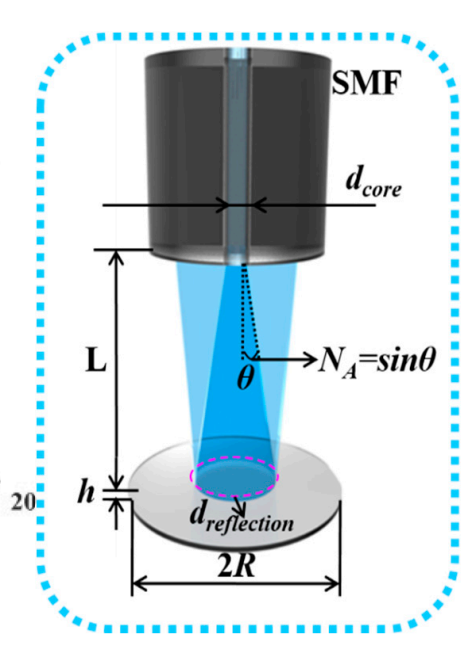

(b)

Figure 2. (a) Natural frequency versus thickness $h$ and radius $R$ of the diaphragm, (b) Schematic diagram for a fiber optic extrinsic Fabry-Perot (FP) interferometer.

Silicon and silicon nitride are commonly chosen as diaphragm materials for fiber optic EFPI sensors because of the mature related processing technology and good mechanical behavior. The normalized central deformation $y(p)$ (deformation of silicon and silicon nitride divided by the maximum deformation of silicon) of these two types of diaphragm at different $R / h$ ratios can be calculated according to Equation (3), as shown in Figure 3, where the natural frequency of the diaphragm and the acoustic pressure are assumed to be 1 . The characteristic parameters of silicon and silicon nitride used in the calculation are given in Table 1. The result shows that the central deformation of a silicon diaphragm is larger than that of a silicon nitride diaphragm for a specific $R / h$ ratio. Consequently, referring to the conclusion mentioned above, when the natural frequency is established, for the same diaphragm size, the deformation of a silicon diaphragm is larger than that of a silicon nitride diaphragm under the same acoustic pressure.

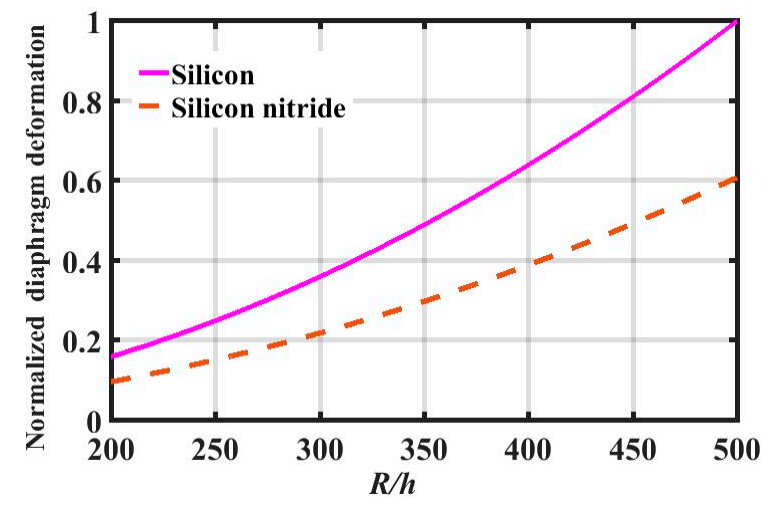

Figure 3. Normalized central deformation $y(p)$ of silicon and silicon nitride diaphragms at different $R / h$ ratios.

Table 1. Characteristic material parameters of silicon and silicon nitride utilized in the design.

\begin{tabular}{cccc}
\hline Material & Poisson's Ratio $\mu$ & Elastic Modulus $E(G P a)$ & Density $\boldsymbol{\rho}\left(\mathbf{k g} / \mathbf{m}^{\mathbf{3}}\right)$ \\
\hline Silicon & 0.22 & 163 & 2330 \\
\hline Silicon Nitride & 0.25 & 310 & 3260 \\
\hline
\end{tabular}


As a result, in this research, a silicon diaphragm with the natural frequency of $60 \mathrm{kHz}$ was designed by employing ANSYS (Ver. 14.5), since the ultrasonic wave in this frequency is widely applied in the industry, defense, and biomedical fields. The diaphragm thickness was $5 \mu \mathrm{m}$, and the diameter was $1120 \mu \mathrm{m}$. In order to accurately control the diaphragm thickness, the MEMS processing technology was applied to a SOI wafer. To define the cavity length during the fabrication of the diaphragm, a deep hole and a shallow hole with different diameters were etched in the handling layer of the SOI wafer, generating a stepped hole with step width of $690 \mu \mathrm{m}$, to accurately position the single mode fiber (SMF). In addition, a commercially available optical fiber PC was employed to replace the customized polished fiber that is often used in most studies.

\subsection{Fabrication}

The fabrication process of the fiber optic EFPI sensors is illustrated in Figure 4. First, a deep hole with diameter of $2.5 \mathrm{~mm}$ and depth of $470 \mu \mathrm{m}$ was etched on the handling layer of the SOI wafer (device layer $5 \mu \mathrm{m}$, box layer $1 \mu \mathrm{m}$, and handling layer $500 \mu \mathrm{m}$, commercially available from Ultrasil Co.) by using a deep reactive ion-etching (DRIE) process (Figure 4a). Then, a smaller hole with diameter of $1120 \mu \mathrm{m}$ and depth of $30 \mu \mathrm{m}$ was etched at the bottom center of the previous deep hole using the spray coating, photolithography, and DRIE processes (Figure 4b). Next, the oxide layer at the bottom of the small hole was completely etched using the buffered oxide etching (BOE) process, and the device layer was used as the self-stopping layer. Finally, a $100 \mathrm{~nm}$-thick gold film was sputtered onto the inner surface of the device layer, behaving as a reflective surface of the FP cavity (Figure 4c). Afterwards, a tube with a slot, functioning as a positioning component for the diaphragm, was manufactured using the 3D printing technology. During the assembly procedure, this tube was fixed in the slot, and the fiber optic PC (type: straight tip, corning: SMF-28e, outside diameter: $2.5 \mathrm{~mm}$ ) was inserted into the tube and then in the deep hole of the diaphragm (Figure 4d). Finally, the fiber optic PC was fixed by using epoxy (353ND) (commonly employed for its long-time reliability). The fabricated sensor probe is shown in Figure 4e.

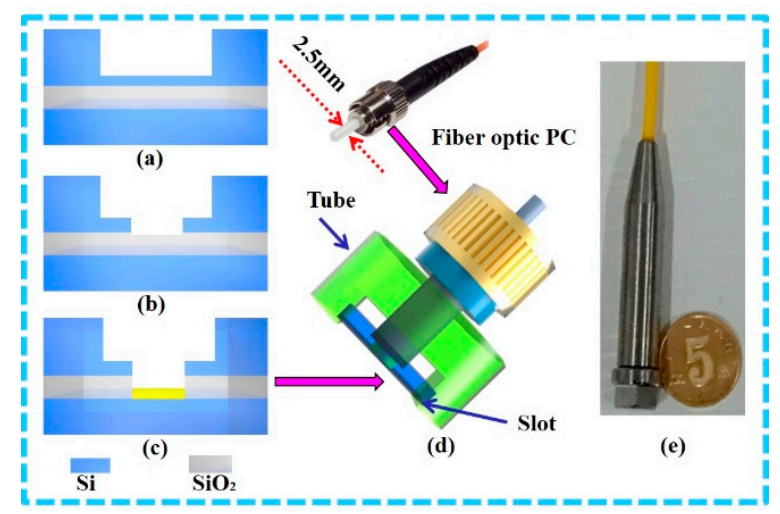

Figure 4. Schematic of fabrication and assembly process of the fiber optic EFPI sensor: (a) Etching of a deep hole on the handling layer of the silicon-on-insulator (SOI) wafer, (b) Etching of a smaller hole in the bottom of the previous deep hole, (c) Removal of the oxide layer and sputtering of the gold film, (d) Assembly of the sensor probe, (e) Optical image of the sealed EFPI sensor.

This procedure presents several advantages: (1) High sensitivity: The thinner the diaphragm thickness is, the higher its sensitivity will be. Therefore, a $5 \mu \mathrm{m}$-thick silicon diaphragm ensures a higher sensitivity than the one obtained so far with other procedures; (2) Low cost: Benefitting from the MEMS fabrication technology, the overall cost of the sensor probe is relatively low, because a single 4 inch SOI wafer can contain hundreds of diaphragms $(\approx 400$, each diaphragm measuring $3.5 \times 3.5 \mathrm{~mm}^{2}$ after scribing). Moreover, the simplified fabrication process can further reduce the cost of the sensor; (3) Easy assembly: The FP cavity length is determined by the DRIE and BOE 
processes, which re controlled by adjusting the process parameters during the first production. The final processed diaphragm and probe (removed from the protection structure) are shown in Figure 5. The stepped hole determines the actual position of the fiber optic PC in the axial direction, defining the cavity length as needed, and thus the assembly procedure is easier and simpler.

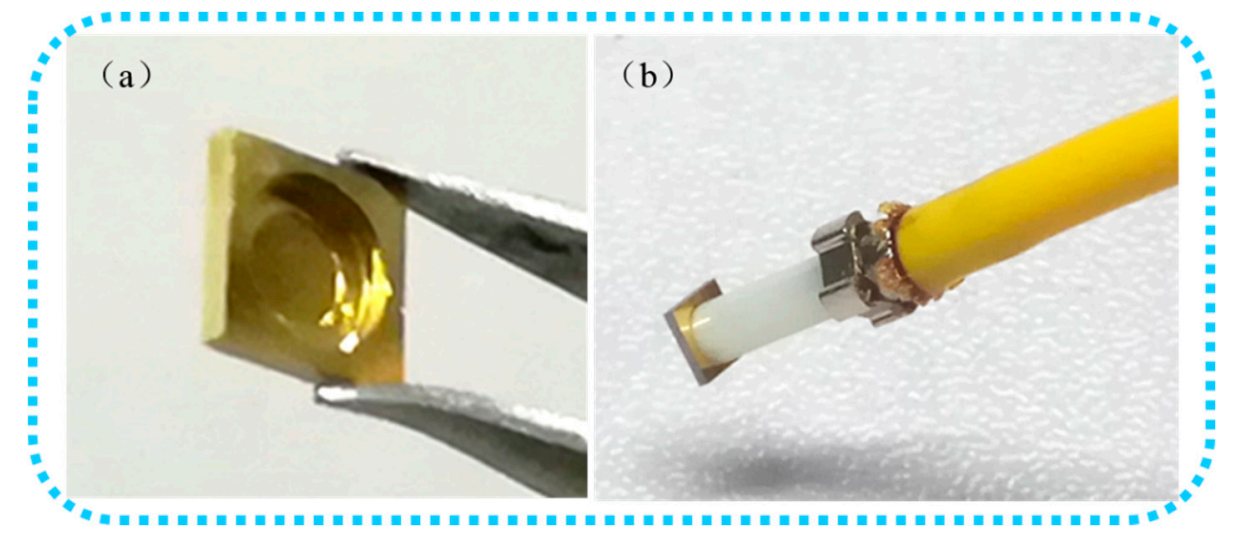

Figure 5. (a) Final processed diaphragm, (b) Final probe (removed from the protection structure).

\section{Experimental Validation}

\subsection{Initial FP Cavity Length}

Figure 6 shows the relationship between normalized intensity and cavity length, as well as the working point designed for the EFPI sensor, which was calculated by the output intensity $I_{1}$ of the FP interference according to Equation (4):

$$
I_{1}=I_{0}\left(R_{1}+R_{2}+2 \sqrt{R_{1} R_{2}} \cos \phi\right)
$$

where $I_{0}$ is the intensity distribution of the light source, $R_{1}$ and $R_{2}$ represent the reflectivity of the optical fiber end surface and the internal face of diaphragm, and $\phi$ is the round-trip phase shift, given by $\phi=4 \pi d / \lambda$ ( $d$ is the FP cavity length, and $\lambda$ is the central wavelength of the light source), and thus the accuracy of the initial cavity length must be at the nanometer scale [17]. The final cavity length in this research was $30.767 \mu \mathrm{m}$, which was calculated by the ratio $\lambda_{1} \lambda_{2} / 2\left(\lambda_{1}-\lambda_{2}\right)\left(\lambda_{1}\right.$ and $\lambda_{2}$ are the adjacent peak wavelengths of the FP interference spectrum). Figure 7 shows the interference spectrum of the FP cavity as well as the superluminescent light emitting diode (SLED) spectrum.

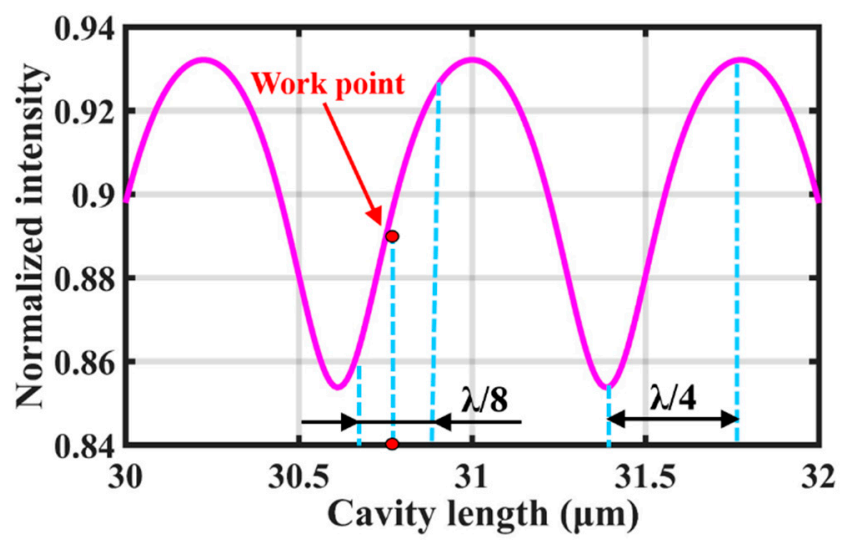

Figure 6. Relationship between normalized intensity and cavity length. 


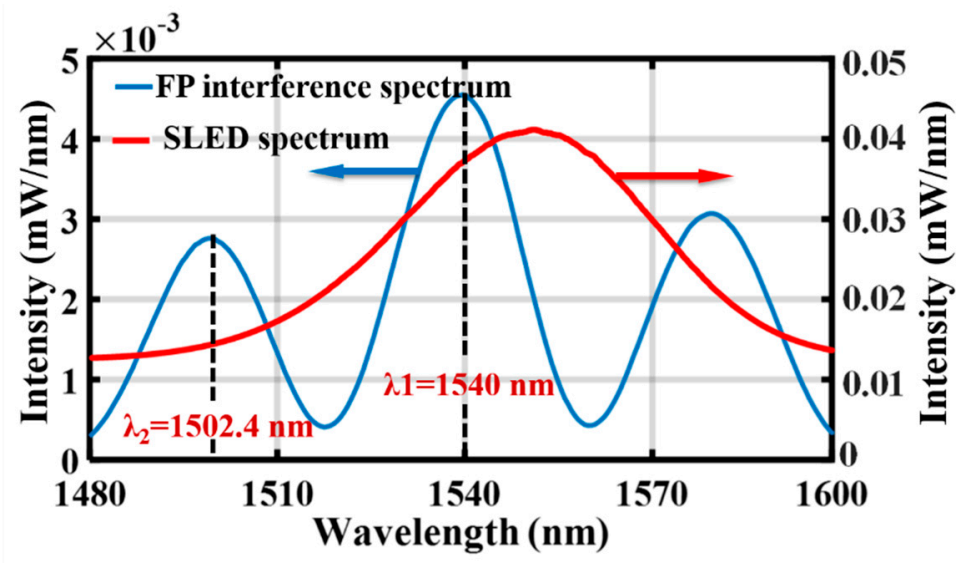

Figure 7. Interference spectrum of the FP cavity together with the superluminescent light emitting diode (SLED) spectrum.

\subsection{Sensitivity and Resolution}

Figure 8 shows the experimental setup employed for the measurement of sensitivity and resolution. It was mainly composed of the fiber optic EFPI ultrasonic sensor, a data acquisition board, a computer, a reference sensor (sensitivity: $5 \mathrm{mV} / \mathrm{Pa}$, amplifier gain: 100, frequency response: 20-70 kHz), and an ultrasonic speaker (frequency: 20-100 kHz). The final testing data were collected by the data acquisition board and processed by the computer. A DFB (distributed feedback) laser (wavelength: $1550 \mathrm{~nm}$, power: $1 \mathrm{~mW}$ ) was used as the light source of the sensing system, and the sensitivity and the amplifier gain of the photodetector were $0.9 \mathrm{~A} / \mathrm{W}$ and $0.6 \times 10^{4} \mathrm{~V} / \mathrm{A}$, respectively. The end reflectivity of the fiber optic PC was $4 \%$, and the insertion losses of the fiber optic and the coupler were less than $0.15 \mathrm{~dB}$.

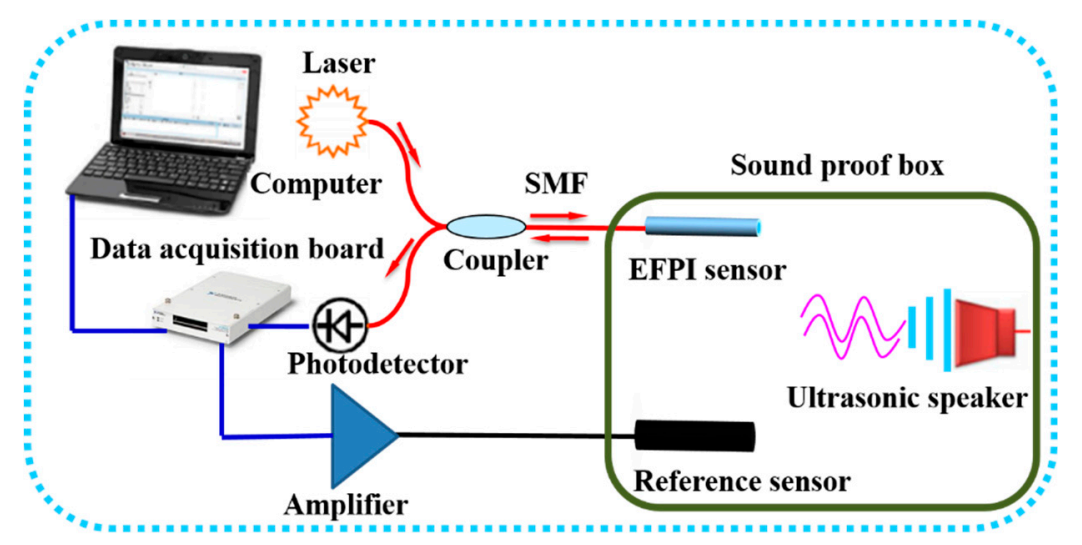

Figure 8. Experimental setup to characterize the fiber optic EFPI sensor system.

The same ultrasonic pressure generated by the ultrasonic speaker was applied to the reference sensor and the EFPI sensor, which were located in a symmetrical position relative to the ultrasonic generator. When the ultrasonic pressure of $40 \mathrm{kHz}$ was applied, the output peak voltages of the EFPI sensor and the reference sensor were $0.8 \mathrm{~V}$ and $0.5 \mathrm{~V}$, respectively, as shown in Figure $9 \mathrm{a}$. According to the sensitivity and amplifier gain of the reference sensor, the ultrasonic pressure applied to both sensors was $1 \mathrm{~Pa}$, and thus the sensitivity of the EFPI sensor was $0.8 \mathrm{~V} / \mathrm{Pa}$. When the ultrasonic pressure was reduced gradually, the minimum output peak voltage of the EFPI sensor was $0.1 \mathrm{~V}$, as shown in Figure $9 \mathrm{~b}$. Since the output signal would be submerged by the voltage noise if the output peak voltage was less than $0.1 \mathrm{~V}$, the minimum detectable pressure of the EFPI sensor for a $40 \mathrm{kHz}$ ultrasonic wave is $0.13 \mathrm{~Pa}$. 


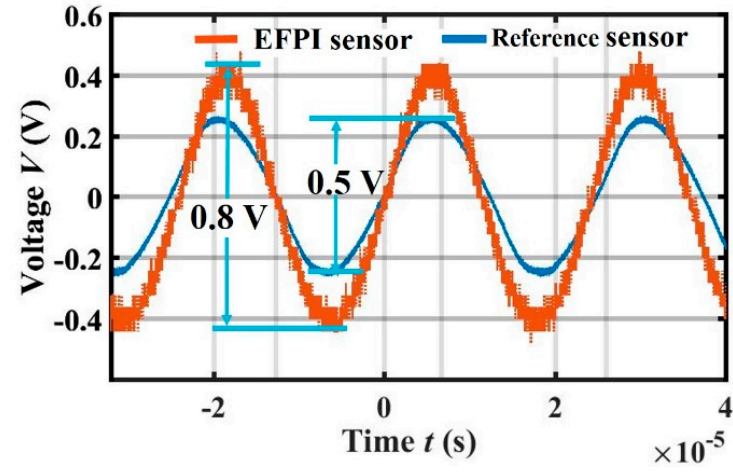

(a)

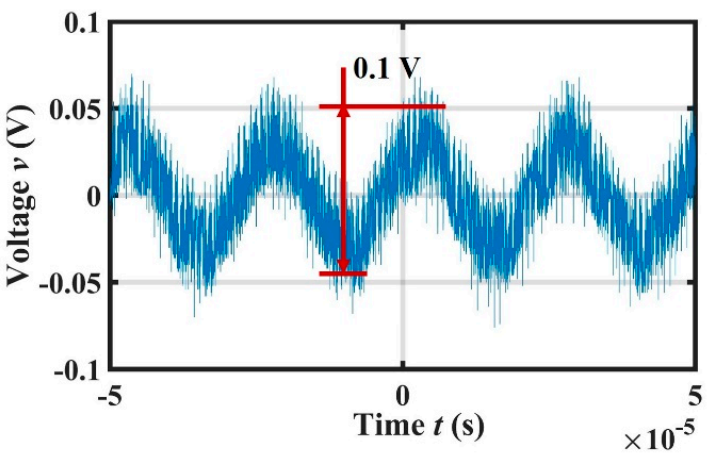

(b)

Figure 9. (a) Output peak voltages of the EFPI sensor and the reference sensor under the same ultrasonic pressure; (b) Minimum output voltage of the EFPI sensor system.

When the output peak voltage was higher than $2.8 \mathrm{~V}$ with increasing ultrasonic pressure, there was a relatively straight line at its valley positions, as shown in Figure 10b. This indicated that the diaphragm deformation exceeded the maximum linear range of the FP interference, and in this region, light intensity changed very little with variations of the cavity length. When weak changes of light intensity could not be recognized by the photodetector, the output voltage became a straight line. As a result, the maximum output peak voltage of the EFPI sensor was $2.8 \mathrm{~V}$, which indicates that the maximum detectable ultrasonic pressure was 3.5 Pa according to the output peak voltage of the reference sensor of $1.75 \mathrm{~V}$, and the linearity of the sensor from $0.13 \mathrm{~Pa}$ to $3.5 \mathrm{~Pa}$ was $3.6 \%$, as shown in Figure 10a.

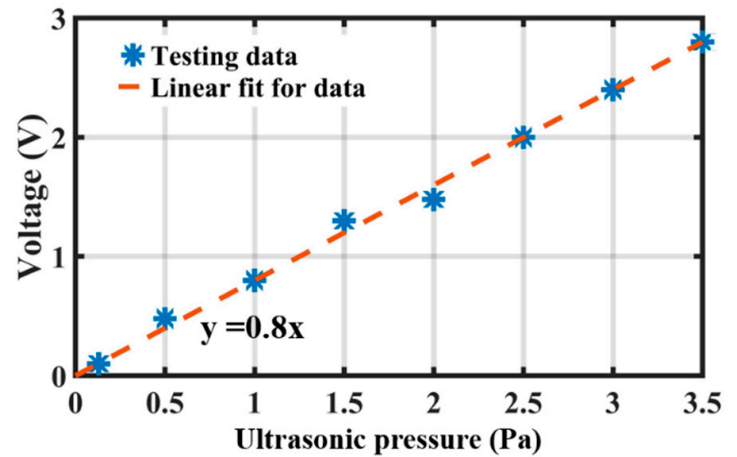

(a)

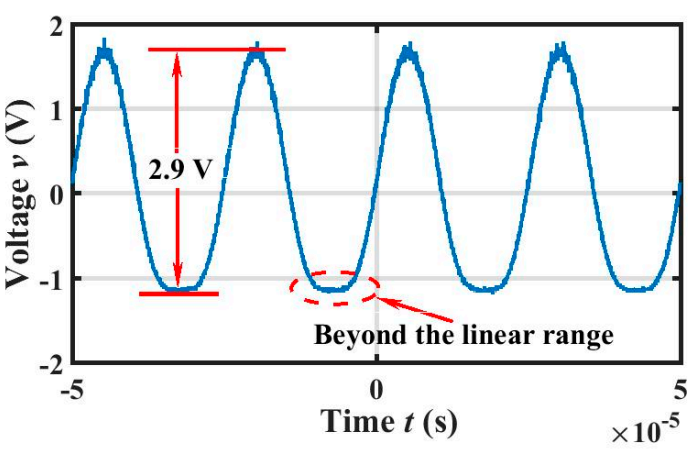

(b)

Figure 10. (a) Linearity of the sensor from $0.13 \mathrm{~Pa}$ to $3.5 \mathrm{~Pa}$; (b) Output voltage of the sensor system when the diaphragm deformation exceeded the maximum linear range of FP interference.

Similarly, the sensor sensitivity versus ultrasonic frequency from $10 \mathrm{kHz}$ to $70 \mathrm{kHz}$ could be obtained by changing the emission frequency of the ultrasonic speaker (since the maximum detection frequency of the reference sensor was $70 \mathrm{kHz}$ ). The results showed that the flatband response was from $20 \mathrm{kHz}$ to $40 \mathrm{kHz}$, and its sensitivity was around $0.8 \mathrm{~V} / \mathrm{Pa}$, as shown in Figure 11a. The resonant frequency was around $64 \mathrm{kHz}$; the small difference between the measured and the calculated resonant frequencies $(60 \mathrm{kHz})$ might have been caused by the slightly different dimensions of the fabricated and modelled diaphragms. Figure $11 \mathrm{~b}$ shows the noise spectral density of the sensor (in $\mathrm{V} / \mathrm{sqrt}(\mathrm{Hz})$ ), which belongs to $1 / f$ noise, and the noise spectral density changes from $1.2 \times 10^{-3} \mathrm{~V} / \mathrm{sqrt}(\mathrm{Hz})$ to $0.5 \times 10^{-3} \mathrm{~V} / \mathrm{sqrt}(\mathrm{Hz})$ between $20 \mathrm{kHz}$ and $40 \mathrm{kHz}$. The minimum detectable pressure can also be given by the noise spectral density of the sensor divided by the sensitivity (in $\mathrm{V} / \mathrm{Pa}$ ). In accordance to this, the minimum detectable ultrasonic pressure was $1.5 \mathrm{mPa} / \mathrm{sqrt}(\mathrm{Hz})-0.625 \mathrm{mPa} / \mathrm{sqrt}(\mathrm{Hz})$ between 
$20 \mathrm{kHz}$ and $40 \mathrm{kHz}$. The background noise of the sensor was dominated by the optoelectronic noise. Therefore, reducing the background noise was a key measure to improve the performance of the sensor. For example, by using a better signal processor such as the one available from FISO Technologies, the performance might be further improved.

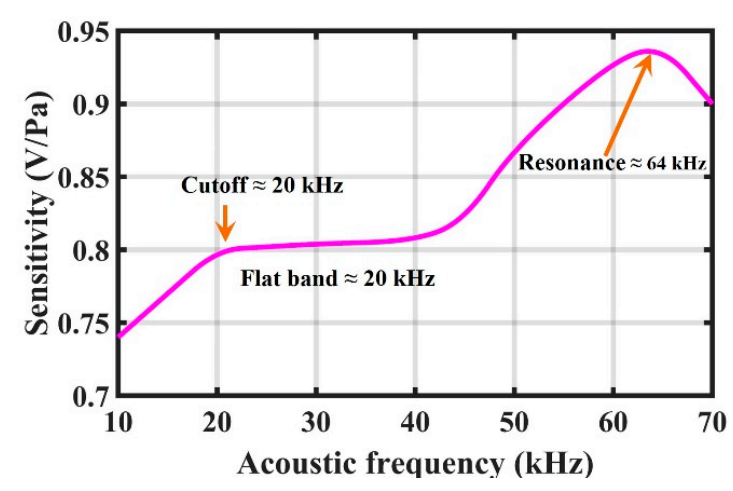

(a)

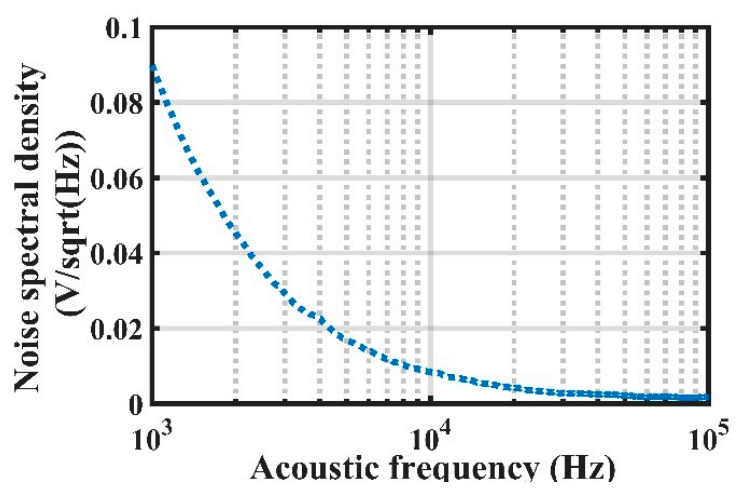

(b)

Figure 11. (a) Sensitivity of the sensor versus acoustic frequency; (b) Noise spectral density of the sensor.

\section{Conclusions}

In this paper, a novel fiber optic EFPI ultrasonic sensor was designed, fabricated, and tested. The diaphragm of the fiber optic EFPI sensor was fabricated by using the MEMS processing technology on a SOI wafer. The diaphragm thickness was $5 \mu \mathrm{m}$, and the difficulty in controlling its desired thickness was greatly reduced by utilizing the proposed manufacturing procedure. In the batch production of the diaphragm, the FP cavity length was determined by a pre-etched stepped hole, which made the sensor assembly easier. Moreover, all the manufacturing processes of the diaphragm were simple and reliable. The described sensor is of relatively low cost, as it benefits from the MEMS fabrication technology and employs cheap and standardized components instead of expensive and customized ones. The final testing result showed that the minimum detectable ultrasonic pressure was $1.5 \mathrm{mPa} / \mathrm{sqrt}(\mathrm{Hz})-0.625 \mathrm{mPa} / \mathrm{sqrt}(\mathrm{Hz})$ between $20 \mathrm{kHz}$ and $40 \mathrm{kHz}$. In addition, the sensing performance can be further improved by choosing a SOI wafer with a thinner device thickness and a further optimized structural design. This flexibility can expand the application fields of fiber optic EFPI ultrasonic sensors.

Author Contributions: H.L. and Y.Y. conceived the idea of this work; H.L. and C.X. proposed the methodology for the measurements; W.S., C.F., and P.Y. made the experimental investigation and data analysis; D.L. helped in checking the whole experimental setup. The manuscript was written by H.L., edited by Y.Y., and revised by all authors.

Funding: We acknowledge the financial support for this research from the National Natural Science Foundation of China (51622509), the Fundamental Research Funds for the Central Universities (No. 3102017jg02007), the 111 Project (Grant No. B13044), and the State Grid Shanghai Municipal Electric Power Company.

Conflicts of Interest: The authors declare no conflict of interest.

\section{References}

1. Gong, Z.; Chen, K.; Zhou, X.; Yang, Y.; Zhao, Z.; Zou, H. High-sensitivity fabry-perot interferometric acoustic sensor for low-frequency acoustic pressure detections. J. Lightwave Technol. 2017, 35, 5276-5279. [CrossRef]

2. Jo, W.; Akkaya, O.C.; Solgaard, O.; Digonnet, M.J.F. Miniature fiber acoustic sensors using a photonic-crystal membrane. Opt. Fiber Technol. 2013, 19, 785-792. [CrossRef]

3. Liu, J.; Yuan, L.; Lei, J.; Zhu, W.; Cheng, B.; Zhang, Q. Micro-cantilever-based fiber optic hydrophone fabricated by a femtosecond laser. Opt. Lett. 2017, 42, 2459-2462. [CrossRef] [PubMed] 
4. Favero, F.C.; Araujo, L.; Bouwmans, G.; Finazzi, V.; Villatoro, J.; Pruneri, V. Spheroidal fabry-perot microcavities in optical fibers for high-sensitivity sensing. Opt. Express 2012, 20, 7112. [CrossRef] [PubMed]

5. Wang, Y.; Wang, D.N.; Wang, C.; Hu, T. Compressible fiber optic micro-fabry-pérot cavity with ultra-high pressure sensitivity. Opt. Express 2013, 21, 14084-14089. [CrossRef] [PubMed]

6. Wenrong, S.; Chenzhao, F.; Delin, L.; Haoyong, L.; Peng, Y.; Yiting, Y. Directional sensitivity of a mems-based fiber-optic extrinsic fabry-perot ultrasonic sensor for partial discharge detection. Sensors 2018, 18, 1975. [CrossRef]

7. Song, L.; Wang, Z.; Wang, A.; Liu, Y.; Cooper, K.L. Angular dependence of the frequency response of an extrinsic Fabry-Perot interferometric (EFPI) fiber acoustic sensor for partial discharge detection. J. Lightwave Technol. 2006, 24, 3433-3438. [CrossRef]

8. Yu, B.; Kim, D.W.; Deng, J.; Xiao, H. Fiber Fabry-Perot sensors for detection of partial discharges in power transformers. Appl. Opt. 2003, 42, 3241-3250. [CrossRef] [PubMed]

9. Pang, C.; Bae, H.; Gupta, A.; Bryden, K.; Yu, M. MEMS Fabry-Perot sensor interrogated by optical system-on-a-chip for simultaneous pressure and temperature sensing. Opt. Express 2013, 21, 21829-21839. [CrossRef] [PubMed]

10. Cibula, E.; Pevec, S.; Lenardic, B.; Pinet, E.; Donlagic, D. Miniature all-glass robust pressure sensor. Opt. Express 2009, 17, 5098-5106. [CrossRef] [PubMed]

11. Akkaya, O.C.; Akkaya, O.; Digonnet, M.J.F.; Kino, G.S.; Solgaard, O. Modeling and Demonstration of Thermally Stable High-Sensitivity Reproducible Acoustic Sensors. J. Microelectromech. Syst. 2012, 21, 1347-1356. [CrossRef]

12. Xia, J.; Xiong, S.; Wang, F.; Luo, H. Wavelength-switched phase interrogator for extrinsic Fabry-Perot interferometric sensors. Opt. Lett. 2016, 41, 3082-3085. [CrossRef] [PubMed]

13. Pevec, S.; Đonlagić, D. Miniature all-fiber Fabry-Perot sensor for simultaneous measurement of pressure and temperature. Appl. Opt. 2012, 51, 4536-4541. [CrossRef] [PubMed]

14. Éric, P. Pressure measurement with fiber-optic sensors: Commercial technologies and applications. Proc. SPIE 2011, 7753, 775304-775308. [CrossRef]

15. Yin, S.; Francis, T.S. Fiber Optic Sensors; CRC Press: Boca Raton, FL, USA, 2002; pp. 54-57.

16. Chenzhao, F.; Wenrong, S.; Haoyong, L.; Delin, L.; Peng, Y.; Yiting, Y. A novel high-performance beam-supported diaphragm structure with enhanced design flexibility for partial discharge detection. Sensors 2017, 17, 593. [CrossRef]

17. Yu, Q.; Zhou, X. Pressure sensor based on the fiber-optic extrinsic Fabry-Perot interferometer. Photonic Sens. 2011, 1, 72-83. [CrossRef] 